\title{
Filtered in / filtered out: Locative media apps and social accessibility in urban spaces
}

\author{
Darryl A. Pieber, The University of Western Ontario, Faculty of Information \& Media Studies, \\ Canada \\ dpieber@uwo.ca
}

\begin{abstract}
Social accessibility involves the capacity of a person to be reached by other people, typically through established social networks. Locative media provide new complications to the issue of social accessibility in urban spaces. They do not connect people with their existing social networks, but rather with people - often strangers - immediately around them. Locative media apps provide users with the ability to manage these anonymous connections through filtering functionalities. This filtering out and filtering in functionality has profound implications for the social relations of strangers in urban spaces. At the heart of the matter is the question of who is made visible and who is made invisible. Many studies of locative media use demonstrate troubling directions in the ways in which they are developed and used. Often, locative media apps reinforce and reify existing racisms and other forms of prejudice, and a general tendency to try to simplify or eliminate differences.
\end{abstract}

\section{Keywords}

locative media, social accessibility, mobile communication, algorithms, filtering, cities, urban, information and communication technologies

\section{Introduction}

Cities have long been a destination point for marginalised people who find they do not or cannot fit in within smaller communities (Fincher \& Jacobs, 1998; Ryan, 2019). This has led to the great diversity characteristic of modern cities, along with corresponding efforts to manage the resultant sensory overload. Simmel (1922 [1955]) proposed the notion of social accessibility as a way of understanding efforts to manage urban social relations. Social accessibility is understood as the ability to be reached by other people (Quan-Haase \& Collins, 2008; Simmel, 1922 [1955]; Weber, Gerth, \& Mills, 1946). The increased use of information and communication technologies has changed the dynamics of social accessibility in urban spaces along with the norms that regulate this accessibility (Milgram, 1970; Simmel, 1922 [1955]; Wellman \& Leighton, 1979; Zerubavel, 1979). People cope with the resulting sensory overload through a series of strategies that help them prioritise and manage social interactions: prioritising relationships, reducing time spent on interactions, blocking unwanted connections (Milgram, 1970; Quan-Haase \& Collins, 2008). The rise in the use of mobile devices has complicated the development of these strategies by making people potentially available anywhere, anytime.

The rise of locative media apps - software programs for mobile smart devices that use the device's location coordinates as a central organising logic for the information that they send and receive (Pieber \& Quan-Haase, in press) - have the potential to profoundly affect the relationship between a city and its inhabitants and among those inhabitants. They have the capacity to challenge efforts to manage social accessibility by providing ad hoc access to nearby strangers, thereby providing users with more information about the people around them than they would otherwise have - or want to have. However, they also offer filtering functionalities and other tools to help manage this increased information. 
In this paper, I examine locative media apps as a communication medium, and the ways in which people can use these apps to manage their social accessibility. At the heart of the matter is the extent to which people are made to be visible or invisible: a person cannot be socially accessible if they are not visible, and people cannot access people they cannot see. While locative media apps have much potential to assist in the relationships between a city and its inhabitants as well as communication efforts among inhabitants, many studies to date demonstrate some concerning trends in the ways in which these apps are made by their developers and deployed by their users. Often, locative media apps reinforce and reify both existing racisms and other forms of prejudice, as well as a general tendency to try to simplify or eliminate differences.

\section{Cities as spaces of difference}

Urban spaces are socially produced. They are not, as Leszczynski (2020) notes, "a priori vacant tabulae rasae” (p. 195). For Lefebvre (1996), there is a symbiotic relationship between a city and its inhabitants. These spaces are produced through the daily mundane movements through the city and the interactions among its diverse inhabitants. These movements and interactions shape the city and in the process the city shapes its inhabitants.

Difference has long been a key characteristic of the modern city. As Fincher and Jacobs (1998) note, this difference manifests through "gender, race, ethnicity, age, life course, sexuality, or any other referent" (p. 5). Furthermore, many people's identities will involve various combinations of these referents. As such, different people will experience the same city differently, even from those immediately around them. This difference is a serious concern for Castells (2002), particularly in fast-growing urban areas. He argues that a lack of a dominant culture to facilitate some degree of assimilation leads to a lack of common vocabulary and values, and therefore no way to communicate with one another and coexist successfully in these large urban areas. He sees within these areas growing fragmentation and alienation from one another leading to an entrenchment away from the communal and the familiar: "I get close to myself, my family, my group, my project, and we split" (p. 555). In this way, he sees communication breaking down altogether within the wider urban environment.

It is not at all clear however that this lack of assimilation is a problem in today's (or tomorrow's) cities. As Tonkiss (2005) notes, this concern about growing alienation and a loss of a sense of community have been common themes in the works of many urban theorists. For Young (1986), this notion of community is typically ill-defined, but she does see some common threads. There is a preference for face-to-face communication among small groups of people in a multitude of situations: work, leisure, family, etc. These groups typically need to be small enough that the members know one another personally. There must be a high degree of commonality.

Tonkiss (2005) sees this "lament" (p. 9) for the loss of community as containing an implicit criticism of urban society. She agrees with Young (1986) that this desire for a return to community is "anti-urban":

\footnotetext{
The idea of community is not simply an antidote to the anonymity of the city; it is a rejection of the urban as a space of strangers, a retreat to familiarity and intimacy as the safest place to be. Such a stance narrows the range of one's concern for others to those who appear familiar or who share similar problems. It stands in difficult relation to the claims of anonymity, to questions of regard between strangers, and to the kind of urban ethics that can bear difference. (Tonkiss, 2005, p. 26)
}

Young (1986) and Tonkiss (2005) recognise the value of face-to-face, close relationships in particular contexts: friendship, cohabitation, family, and so on. However, they both suggest that this approach is inappropriate as a means to examine urban social relations overall. They argue that urban social relations are relations among 
strangers. Rather than face-to-face interaction, the urban social relations of strangers are characterised by "side-by-side" relations that take place in public spaces such as parks, streets, bars, cultural venues, etc. Young (1986, p. 21) notes that "in such public spaces, the diversity of the city's residents comes together and dwells side by side, sometimes appreciating one another, entertaining one another, or just chatting, always to go off again as strangers".

Tonkiss (2005) suggests that there exists among strangers in urban environments an indifference to difference. She contends that, "alongside an active politics that recognises differences...there lies an ordinary urban ethics that looks straight past it" (Tonkiss, 2005, p. 10). While admittedly "fragile, grudging, uneven" (p. 10), this indifference can afford people some space in which to express individual difference. As Urry notes, urban spaces afford the possibility for differing bodies to find a space:

The modern city gives room to the individual and to the peculiarities of their inner and outer development. It is the spatial form of modern urban life that permits the unique development of individuals who socially interact with an exceptionally wide range of contacts. (Urry, 2007, p. 23)

\section{Sensory overload and social accessibility in the city}

For Simmel (1997), “[t]here is perhaps no psychic phenomenon which has been so unconditionally reserved to the metropolis as has the blasé attitude. The blasé attitude results first from the rapidly changing and closely compressed contrasting stimulations of the nerves" (p. 178). The sheer volume of strangers with whom urban dwellers come into daily contact results in the reduction of both the quantity and the quality of social interactions: they engage in fewer interactions with strangers than may be the case in small towns and rural environments, and these interactions tend to be considerably more superficial, so as to be able to manage the sensory overload that arises from the larger populations of urban spaces.

Simmel's (1922 [1955]) almost 100year-old concept of social accessibility still is a useful tool to investigate the ways in which urban dwellers navigate the information overload brought on by the significant increase in the number and diversity of people encountered daily in cities. Social accessibility is the ability to be reached by other people (Quan-Haase \& Collins, 2008; Simmel, 1922 [1955]; Weber, Gerth, \& Mills, 1946). Milgram (1970) established 50 years ago that urban dwellers manage social accessibility by restricting moral and social interactions with others. He observed that urban social relations are often interpreted as being considerably colder and ruder than in rural settings (Milgram, 1970). However, he proposes an alternative interpretation:

\footnotetext{
A rule of urban life is respect for other people's emotional and social privacy, perhaps because physical privacy is so hard to achieve. And in situations for which the standards are heterogeneous, it is much harder to know whether taking an active role is unwarranted meddling or an appropriate response to a critical situation. (Milgram, 1970, p. 1463)
}

In addition to serving as a coping strategy for stimulus overload in urban spaces, this "respect for other people's emotional and social privacy" can facilitate the indifference to difference that Tonkiss (2005) identifies as essential to a functioning, heterogeneous city.

Quan-Haase and Collins (2008) contend that the concept of social accessibility takes on a greater relevance in the study of urban social relations with the rise in information and communication technologies (ICTs) such as e-mail, instant messaging and social networking. Such ICTs greatly expand the ways in which people can be reached, irrespective of time or distance. In this way, they pose new challenges to urban dwellers' efforts to manage their social accessibility among their networks of connections (friends, family, colleagues, and so on). The increased use of information and communication technol- 
ogies has changed the dynamics of social accessibility in urban spaces along with the norms that regulate this accessibility (Milgram, 1970; Simmel, 1922 [1955]; Wellman \& Leighton, 1979; Zerubavel, 1979). And, as Quan-Haase and Collins (2008) point out, these new challenges are even more pronounced for urban youth: numerous studies have documented both the greater likelihood of urban youth to adopt these technologies and a greater sense of pressure to be always accessible. The rise in popularity and use of locative media further complicates the management of urban social accessibility by introducing potential ephemeral, ad hoc connections with nearby strangers.

\section{What are locative media?}

Locative media are a set of ICTs - hardware and software - for mobile smart devices (smartphones, tablets, etc.) that make use of the device's location coordinates as a central component of their operations. On the software side, locative media apps are software programs for mobile smart devices that rely on the device's location coordinates to send and receive information that is relevant to the specific location where the device is, at the specific moment that it is there (Pieber \& Quan-Haase, in press). As Wilken (2019) notes, there has been a significant rise in the use of a device's location coordinates by a wide variety of mobile apps on the market:

Every day, tens of millions of mobile users identify and register their location in various ways via the functions embedded within or downloaded onto their mobile devices. For example, the images and videos we tag, upload, and share to sites such as Snapchat, Instagram, WhatsApp, Flickr, Facebook, and Kakao, are now typically geocoded. Positional information is also a core component of dating and hook-up apps. (Wilken, 2019, p. 7)

A number of scholars (Sutko \& de Souza e Silva, 2011; Wilken, 2019; Wilken \& Goggin, 2015) include under the umbrella of locative media any mobile app that makes use of the device's location coordinates. In this instance, geotagging photos on Instagram or checking in on Facebook constitute locative media functionality. However, I am interested in a much narrower understanding of locative media. In this paper, I consider those mobile apps that make use of the device's location coordinates as their central organising principle, that is, those apps which present to their users information about their immediate surroundings. This information can be about points of interest that are nearby (restaurants, tourist attractions, entertainment, and so on). It can also be information about the people in their immediate vicinity. As well, it can be about navigating and travelling through a city. Examples of locative media apps include information sharing apps like Yelp; dating apps like Tinder and Grindr; mobile gaming apps like Pokémon Go; navigation apps like Google Maps and ride-sharing apps like Uber and Lyft. By contrast, a weather app is not a locative media app in this understanding of the term because it provides weather information from the nearest weather station rather than from the specific location where the app user is standing (Pieber \& QuanHaase, in press).

These types of mobile apps directly mediate between their users and the people and spaces immediately around them (Leszczynski, 2015). As such, they evoke a profound shift in the way we understand ICTs - from "anytime anywhere" computing to "right here and right now" computing (Kitchin, 2014; Villi, 2015). Where traditional ICTs require us to enter a virtual world to access generic information, locative media attach information to specific spaces at specific times, thereby augmenting a user's experience of the physical world with information that is specific to the space and time the user is occupying (McCullough, 2006). For Farman (2012), locative media bring together the virtual and physical worlds into a new, hybrid space. However, this notion of a hybrid space risks ignoring a key characteristic of locative media: its central organising principle is physical space. Locative media users do not go to some other space to ac- 
cess the information in the way that virtual reality users might. Rather, as Leszczynski (2015) proposes, locative media users access digital information in the physical space where they are standing. The information is anchored to the physical space.

Locative media apps complicate Lefebvre's (1996) notion of the symbiotic relationship between a city and its inhabitants by adding another participant in the production of urban space. Kitchin and Dodge (2011) have coined the term code/space to describe the role of locative media in the production of urban space whereby "software and the spatiality of everyday life become mutually constituted, that is, produced through one another" (p. 16). As I explore in this article, this third wheel in the symbiotic relationship has the capacity to affect this relationship in significant ways. It can help render parts of the city visible or invisible to some or all of its inhabitants. Similarly, locative media apps can render the inhabitants of the city visible or invisible to other inhabitants. In this way, it can have profound effects on people's social accessibility.

An additional important feature of locative media apps is that they tend to mediate connections among strangers. For example, while it is possible to come across the profiles of friends and family members on hook-up apps like Tinder, this is not their main purpose. As well, information sharing apps like Yelp rely on ratings and other data that is crowdsourced by their many users (Frith, 2017; Sutko \& de Souza e Silva, 2011). In this paper, I look specifically at locative media use in urban spaces in large part because of this mediation among strangers. As discussed earlier, social relations in cities tend most often to be among strangers. Locative media apps can connect nearby strangers with one another, as well as provide information about unfamiliar surroundings that urban users may find themselves in (McCullough, 2006).

\section{Locative media and social accessibility}

As mentioned earlier, ICTs present significant challenges to urban dwellers' ability to manage their social accessibility because they make it possible to be reached anywhere anytime, thereby greatly increasing the potential for sensory overload. This increase in accessibility, however, is largely from existing social networks: friends, family, co-workers, and so on (Quan-Haase \& Collins, 2008). Locative media apps further complicate the issue by making people potentially reachable by nearby strangers. And as people move through the city, they become reachable by different strangers - the ones they pass along their way. Locative media apps can increase awareness of these strangers by increasing the amount of information available, turning strangers passing one another on the street into what Licoppe (2016, p. 108) calls pseudonymous strangers: people "with whom one may never have interacted or talked about before [...] but who are not complete strangers either, for the locative app usually makes available some info about them". Conversely, as Sutko and de Souza e Silva (2011) point out, locative media apps can be deployed to help manage users' social accessibility. Most apps have some degree of filtering functionality which allows users to establish parameters around which strangers' information they see, and which strangers can in turn see their information. When deployed effectively, these apps have the potential to facilitate mutually sought-after serendipitous connections with nearby strangers. However, when deployed less than optimally, they can contribute to the already overwhelming stimuli of the urban spaces they are passing through. And, as I discuss below, they can reinforce existing racisms and other prejudices. As locative media apps increase in popularity and use (Wilken, 2019), Licoppe (2016) warns about the implications of a city full of people accessing and sharing their location and other personal information through these apps. The sophistication and flexibility of apps' filtering capabilities will 
become of great importance in helping to manage the sensory overload that these apps can bring on in a way that can still allow for a city's diversity and city dwellers' indifference to difference.

\section{Locative media apps and (in) visibility in the city}

Visibility and invisibility play a pivotal role in social accessibility. In order to be accessible, people must be able to be aware of who else is around them. Thus, the filtering capabilities of locative media apps can play a significant role in an urban dweller's social accessibility. This is because locative media apps can make people visible through their interface in a physical space where they might not otherwise be noticed. They can in turn make people invisible through their interface in a space that they are physically present in. As people come to rely more and more on locative media apps to navigate through cities, who and what is visible (or not) through the apps' interfaces will come to have an increasingly important role in how people perceive the urban spaces they move through, and who they can and cannot see in those spaces.

\subsection{Making people visible}

Locative media apps have the capacity to make people visible in spaces where they might otherwise go unseen. In a study (Blackwell, Birnholtz, \& Abbott, 2014) of users of Grindr, a dating app for gay men, participants remarked that the app made gay men visible to one another almost anywhere in the city where they used the app. This awareness of other LGBTQ+ people nearby can have the effect of making them feel safe in spaces that may otherwise feel uncomfortable or even threatening. As Roth (2014) observes, locative media dating apps like Grindr and Scruff (another dating app, targeted at gay men who self-identify as "bears") complicate the identification of spaces as either gay or straight:
Concerns over whether spaces are hetero- or homosexual - and, consequently, whether those spaces can be "reterritorialised" as queer - neglects the possibility of heterogeneous spaces in which queerness can coexist, omnipresently, with heterosexuality [...] Queerness does not need to be subsumed by heterosexuality for the two to occupy the same physical space when queer communities can exist, invisibly but to their participants, on mobile phone screens. (Roth, 2014, p. 2127)

In making LGBTQ+ people visible to one another throughout an urban space, locative media apps like Grindr and Scruff can also reveal a queer map of the space that is layered over and anchored to the physical space, visible for those with the eyes (or apps in this case) to see it.

However, this visibility is not without its risks because these apps are not only available for use by people who identify as either LGBTQ+ or friendly toward this population. Anyone can download and use gay-targeted locative media apps and view the queer map of the city and its LGBTQ+ inhabitants. In a study by Albury and Byron (2016), participants identified being "outed" to the wrong people in the wrong spaces as being one of the biggest risks for them in using gay-targeted locative media apps. Hjorth, Pink, and Horst (2018) found similar concerns in their study of lesbian couples' use of locative media. In their study, participants expressed varying degrees of comfort and discomfort in their use of locative media as it concerned broadcasting aspects of their sexuality. Where this information was broadcast widely through these apps, participants often saw this act as "political [...] as a form of everyday activism" (p. 1217).

Visibility through locative media apps thus presents significant implications for social accessibility in urban spaces. Indeed, it is a prerequisite for social accessibility: one who is invisible cannot be reached and conversely one cannot reach people who they cannot see. Prior to the introduction of locative media apps targeted at LGBTQ+ populations, members of this community would have to find each other in designated spaces in the city - gay 
bars, gay neighbourhoods, and so on - or by using a series of established codes and cues (Crooks, 2013). These locative media apps open up the whole city as a possibility for social accessibility among LGBTQ+ people, but this new openness comes with risks and requires new strategies for negotiating social accessibility to avoid unwanted (and potentially dangerous) attention.

\subsection{Making people invisible}

Locative media apps have the capacity to make people invisible in spaces where they are physically present. Locative media apps typically provide users with ways to filter the information they see, usually based on some set of personal interests. Grindr, for example, allows gay men to set filters based on the types of men they are interested (or not interested) in meeting (Conner, 2019). Tinder, by contrast, requires that both users "swipe right" on respective profile pictures in order to connect with one another (David \& Cambre, 2016). Such functionalities are essential tools for the management of social accessibility: they help users make themselves visible or invisible to other people (and to make other people visible or invisible to users). However, these functionalities are not without their consequences.

Grindr's filtering functionality makes it possible for users to filter people out based upon identity markers such as race, age, body type and so on. While filtering is an essential tool in the management of urban sensory overload through social accessibility, the way in which it manifests in apps like Grindr has been widely criticised for entrenching existing prejudices into the app. ${ }^{1}$ And, while these prejudices already exist in the offline world, these filters become in a way more insidious because the filtering is invisible

1 In June of 2020, Grindr announced that it would be removing its ethnicity filtering functionality as a response to the Black Lives Matter protests taking place throughout the United States and other countries. As of the writing of this paper, however, the ethnicity filter is still present and functional within the app (Hunte, 2020). to those being filtered out (Conner, 2019; Mason, 2016). As one participant in Conner's (2019) study remarks: "Users can just select me out of the equation. When I look at my White friends' Grindr accounts they have messages in the hundreds. I can barely even get a single message. I feel sometimes as if I don't exist" (p. 403). In addition to this participant's difficulties in connecting with other gay men through the app, the erasure he experiences also serves to reduce - or even eliminate - any sense of security he may feel though being able to locate other LGBTQ+ people in unfriendly spaces (as discussed earlier) because he has been blocked from seeing them.

Frith (2017) introduces another dimension to the problem of invisibility through locative media apps. He discusses the situation in Denton, a city in Texas where the city's entire Mexican neighbourhood is all but invisible through locative media information sharing apps (what he calls spatial search apps). He also discusses the near total absence in locative media apps of an African American neighbourhood in Pittsburgh, Pennsylvania, known as The Hill. These two examples demonstrate a reinforcement in the apps of historical racial divides in the two cities. But they also point out a case of digital divide: the people who frequent the bars, restaurants and other venues in these two neighbourhoods tend not to have smart devices and/or tend not to make use of locative media apps such as Yelp. Given the rise in popularity of locative media apps (Wilken, 2019), and the accompanying reliance on them to navigate both the city and social accessibility in the city, such absences serve to entrench and reify historical segregations, and confound efforts to achieve the indifference to difference that Tonkiss (2005) identifies as essential to the navigation of diversity in urban spaces.

\section{Algorithms of (in)visibility}

Given that the information available through locative media apps is largely crowdsourced, it can be argued that much of the responsibility for how these apps 
work rests with those people who use them and indirectly with those who do not. However, locative media apps - like all software applications - have underlying logics that guide how they work. These logics are coded into their algorithms.

At the most basic level, algorithms are collections of computer programming code that work together to provide sets of instructions to platforms on how to accumulate, process and manipulate the data they gather. But as Finn (2017, p. 5) notes, algorithms are much more than this basic definition would suggest: "they are also bodies of knowledge, sets of rules and procedures, that can be implemented in practice. They are technical entities that have their own existence independent of their human practitioners, but which operate through the medium of culture". Such complications in the understanding of what algorithms are and how they work often go unacknowledged, because they are assumed to operate "objectively." As Chander (2017, p. 1034) notes: "Algorithms can make decisionmaking seem fair precisely because computers are logical entities which should not be infected by all-too-human bias. But that would be an unwarranted assumption".

While a computer engineer may argue that the code within algorithms is unbiased, "a pinnacle of Enlightenment, rationalist thought" (Finn, 2017, p. 7), they in fact have biases - intended and unintentional - encoded into them.

\subsection{Encoding biases}

Ruha Benjamin (2019) takes an extensive look at the ways in which biases are encoded into algorithms. While she is primarily concerned with racial bias, she notes that other identifications such as class, gender, and so on, interact with and compound instances of algorithmic bias. She identifies three main ways in which these encodings occur. The first involves programmers encoding their own deeply held biases. While the programmers may be unaware of these biases because they are so deeply engrained, Benjamin (2019, p. 28) argues that the encodings themselves cannot be considered to be unconscious or unin- tended because "there is no way to create something without some intention and intended user in mind". Programme designers have a target audience in mind when developing their programmes. The characteristics of these target audiences inform the design of the programme. A second way in which existing biases can be encoded into programmes is through the programmes' processes of "deep learning”. Programmes increasingly absorb data from a vast array of existing sources to fine tune their processes. In this way, they incorporate the existing biases of the people who have contributed the data. As this process of deep learning unfolds, programmes become increasingly more complicated and, as Chander (2017) observes, even the programmes' developers may end up not knowing exactly what the programmes are doing. A third way in which these encodings can occur is through a lack of recognition of the social and historical contexts in which the programme will operate. If existing biases are not examined and accounted for, they will be replicated and reinforced in the programme code (Benjamin, 2019).

We can see these encodings at work in Grindr's filtering functionality as discussed earlier. The ability to filter out users based upon their ethnicity and other identifications has the effect of making people invisible in the app even though they are present in a space. This encoding is not an accident - the app is meant to work in this way. The app has been coded to meet the needs of their target audience. As noted earlier, these biases already exist in the gay male community where, as Mason (2016) notes, racism (and other forms of prejudice) masquerade as personal preference. The app's developers do not seem to have considered the social and historical contexts in which the app will be used, as Benjamin (2019) argues for.

\subsection{Erasing difference}

As Veel and Thylstrup (2018) point out, locative media algorithms are, in the background, forever classifying users and data into distinct categories, and identifying who and what to include and exclude. 
These processes can have the effect of discouraging or altogether erasing differences that cannot or will not fit neatly into the defined categories. A case in point is the gay dating app Scruff. Scruff is targeted at gay men who self-identify as "bears". This term is intentionally difficult to define. As Roth (2014) notes, the term "originally emerged as a form of resistance to the 'body fascism' of American gay culture in the mid- $20^{\text {th }}$ century. In the case of bearishness, this was encapsulated in the feeling that 'You can't be gay. You're too old, hairy, and fat'” (pp. 2124-2125). The category "bear" has historically been intentionally left as an empty signifier, as a rejection of the restrictive norms of mainstream gay male society in the United States. But despite this intentional ambiguity around body image, Scruff encourages its users to slot themselves into one of several categories of bear that its developers have established. While this self-categorisation is not mandatory, those who do not categorise themselves risk becoming invisible in the app if other users choose to filter their searches based on the categories. In this way, Scruff restricts the potential diversity of body expressions possible among bears. And those that do not or cannot slot themselves into one of the app's predefined categories risk being made invisible to other members of their community (Roth, 2014). Such efforts to simplify or eliminate the complexity of differences on display in urban spaces are, to borrow Young's (1986) term, anti-urban.

\section{3 (In) visibility}

A key concern for Benjamin (2019) involves an algorithm's ability to expose marginalised people to systems of surveillance and corresponding policing of behaviours and ways of being. She (2019, p. 99) usefully compares this act of exposure to the processing of film, which she describes as "a delicate process - artful, scientific, and entangled in forms of social and political vulnerability and risk". Like the art of film processing, negotiating visibility and invisibility - and social accessibility through the algorithms of locative media apps (and other technologies) is fraught for marginalised people.

On the one hand, to be absent - invisible - within these apps is to risk being further marginalised. A major problem with locative media apps in this regards seems to be its reliance on user-generated data, as we see, for example, in Frith's (2017) examination of Denton's Mexican neighbourhood and The Hill neighbourhood in Pittsburgh: Yelp lacks data about these neighbourhoods because users of the app are not adding it. However, we can also see the effects of intentional coding that causes marginalised people to become invisible in the gay dating apps Grindr and Scruff. Filtering functionality has been added to both to facilitate the erasure of some people as discussed earlier (Conner, 2019; Roth, 2014).

On the other hand, to be visible within locative media apps can bring many potential risks. For Benjamin (2019), a key concern of visibility for Black people (and other marginalised groups) involves the surveillance potentials that these technologies can afford. As she says, "a key feature of Black life in racist societies is the constant threat of exposure and of being misread; and that being exposed is also a process of enclosure, a form of suffocating social constriction" (Benjamin, 2019, p. 101). And this is equally true for LGBTQ+ people where the threat of exposure - and even being read as LGBTQ+ - can bring serious physical and other dangers (Albury \& Byron, 2016; Hjorth, Pink, \& Horst, 2018). This concern is particularly relevant for locative media apps which, by their nature, can track people's movements through space in real time, and can keep a record of places they've visited.

The issues of visibility and invisibility of marginalised people through locative media apps are thus not straight forward. There are many ways in which biases are encoded into the apps' algorithms, thereby perpetuating and potentially exacerbating already existing discriminations against marginalised people. As Benjamin (2019, p. 99) observes: "Who is seen and under what terms holds a mirror onto 
more far-reaching forms of power and inequality".

\subsection{Reading the glitch}

As Chander (2017) points out, the increasing complexity of algorithms makes it difficult if not impossible to examine biases encoded into the algorithms themselves. This situation is further complicated by the fact that many algorithms driving locative media and other apps and internet services are typically proprietary. As a result, the companies that hold the patents on them are unlikely to be willing to make them available to public scrutiny. Chander instead argues that we should pay attention to both the inputs (the data that algorithms process) and the outcomes of algorithmic processing. Benjamin (2019) and Leszczynski (2020) both argue for an examination of the "glitches" that are found in these systems as a fruitful approach to identifying inherent problems. At its simplest level, a glitch is an instance where a technological system does not operate the way its developers or users expect it to. But Benjamin and Leszczynski argue that glitches are in fact much more than this. For Benjamin, glitches can act as a "technological canary in the coalmine" (Benjamin, 2019, p. 47) that indicate biases encoded into the algorithm:

Glitches are generally considered a fleeting interruption of an otherwise benign system, not an enduring and constitutive feature of social life. But what if we understand glitches instead to be a slippery place [...] between fleeting and durable, micro-interactions and macro-structures, individual hate and institutional indifference? Perhaps in that case glitches are not spurious, but rather a kind of signal of how the system operates. Not an aberration but a form of evidence, illuminating underlying flaws in a corrupted system. (Benjamin, 2019, p. 80)

In short, Benjamin (2019) argues, when algorithmic outcomes (re)produce biases, the algorithm is working exactly as its designers intended.

Both Benjamin (2019) and Leszczynski (2020) also see glitches as potential sites of resistance. For Benjamin, this potential for resistance begins with a recognition and rejection of the complex ways in which algorithms contribute to a process of dehumanisation. She goes further, though, in arguing that it is not enough simply to reject biased systems. It is essential as well to begin the process of imagining new ones. Leszczynski argues for bringing a "feminist politics of the urban everyday" (Leszczynski, 2020, p. 191) to understanding systems and their algorithms. She wants to examine questions of biases that manifest (or are dismissed) as glitches from the perspective of those experiencing the biases and glitches. Rather than understanding glitches as failures of the system to operate, she understands them as potential "opportunities for mundane digital tactics to negotiate, divert, diffract, or differently assemble" the interfaces between system and user in ways that are "inherently and immediately political” (Leszczynski, 2020, p. 201).

Frith's (2017) discussion of the Denton and Pittsburgh neighbourhoods that are not visible on the Yelp app provides a useful subject for considering this notion of the glitch as potential warning of systemic bias and possibly as site of resistance. Frith rightly identifies these absences in Yelp's information offerings as being evidence of a systemic replication of existing biases in the physical structuring of both cities. As he notes, deciding which locations, streets, neighbourhoods, and so on, are represented on a map have serious consequences for how a space is navigated and understood by those moving through it. Even though locative media mapping apps like Yelp put the decisions about what to include and what not to include largely in the hands of their users, this power to define a space and how it will be read is still not available to everyone. As Frith (2017, p. 543) notes, some people are excluded "because they do not have access to the right technologies or the inclination or digital literacies necessary to contribute to community mapping projects. Research has also shown that only a small minority of users actually create spatial content for location-based services". As Kitchin 
and Dodge (2011) point out, locative media apps are increasingly implicated in the production of urban spaces as a third player in the symbiotic relationship between a city and its inhabitants (Lefebvre, 1996). This invisibility of the Hispanic inhabitants of Denton and the African American inhabitants of Pittsburgh means that they have no say in the way in which their respective cities are mapped and thereby navigated and understood. The absence of these neighbourhoods on Yelp reinforces the already existing physical divides in the respective cities, and limits possibilities for interactions between the residents on either side (Frith, 2017).

But could there be other ways to read these glitches? Given the systems of surveillance that people of colour are already subject to on a daily basis (Benjamin, 2019), could it be that the residents of these neighbourhoods understand locative media mapping apps as yet another layer of surveillance? Could it be that the residents of these neighbourhoods have alternative tactics for navigating through their cities in use that better serve these residents' needs and avoid the increased layer of surveillance from locative media apps?

The algorithms of locative media apps are heavily implicated in the ability of city dwellers to be visible or invisible in urban spaces, thereby affecting their social accessibility with other inhabitants of their city. These algorithms can and do replicate and often exacerbate existing biases against some people based on various identifications such as race, ethnicity, gender, sexual orientation, and so on. But issues of visibility and invisibility are not straightforward. The ability to be visible in a space is not always desirable, but nor is invisibility. Benjamin's (2019) notion of the glitch as a warning signal indicating systemic racism, together with Leszczynski's (2020) notion of the glitch as potential site of resistance provide useful tools for interrogating the ways in which the algorithms of locative media apps contribute to users' (in)visibility in urban spaces and their ability to navigate social accessibility in and through the city.

\section{Conclusion}

Cities have long been characterised by difference, leading to great potential for sensory overload through the vast increase in the numbers and variety of people, places, information and other sources of stimuli. Urban dwellers develop coping strategies to manage this sensory overload, predominantly by filtering most of it out. To deal with the vast quantity and variety of social interactions that an urban dweller is exposed to daily, urban dwellers engage in a careful management of their social accessibility, understood as the ease with which they can be reached (Simmel, 1922 [1955]). Urban dwellers develop an indifference to the great differences (Tonkiss, 2005) in terms of ethnicity, gender, sexuality and other referents that exist side-by-side every day in urban spaces.

Locative media apps present a range of opportunities and challenges to the successful management of social accessibility. These apps can provide additional information about nearby strangers that can challenge the anonymity among strangers that is at the core of urban indifference to difference. However, locative media provide filters and other tools to their users to help them manage these new challenges to their social accessibility (Wilken, 2019). But careful consideration must be given to the ways in which these tools are programmed by locative media app developers, and the ways in which they are deployed by app users. The visibility and invisibility afforded by these tools lay at the heart of social accessibility management: one cannot be socially accessible if one is not visible; and, one cannot access others one cannot see.

Many studies (Albury \& Byron, 2016; Blackwell, Birnholtz, \& Abbott, 2014; Conner, 2019; Frith, 2017; Mason, 2016; Roth, 2014) of locative media app use demonstrate disturbing trends toward the over-simplification of difference among the urban dwellers using the apps. These studies also demonstrate a tendency among users to filter out differences and, in the process reinforcing and reifying existing racisms and other prejudices that al- 
ready exist in the offline world. These acts of filtering are arguably more insidious than the offline prejudices in that the erasures being enacted are not visible to those being filtered out.

While locative media apps show great potential to mediate relations between cities and their inhabitants - and among those inhabitants (McCullough, 2006) the underlying logics being programmed into them by some locative media app developers, and the ways in which the apps are being deployed by users often seek to diminish or erase altogether any visible sign of the diversity of a city. In this way, locative media apps become decidedly anti-urban.

\section{References}

Albury, K., \& Byron, P. (2016). Safe on my phone? Same-sex attracted young people's negotiations of intimacy, visibility, and risk on digital hook-up apps. Social Media + Society, 2(4), 1-10.

Benjamin, R. (2019). Race after technology: Abolitionist tools for the New Jim Code. Medford: Polity.

Blackwell, C., Birnholtz, J., \& Abbott, C. (2014). Seeing and being seen: Co-situation and impression formation using Grindr, a location-aware gay dating app. New Media \& Society, 17(7), 1117-1136.

Castells, M. (2002). Local and global: Cities in the network society. Tijdschrift voor Economische en Sociale Geografie, 93(5), 548-558.

Chander, A. (2017). The racist algorithm. Michigan Law Review, 115(6), 1023-1046.

Conner, C. T. (2019). The gay gayze: Expressions of inequality on Grindr. The Sociological Quarterly, 60(3), 397-419.

Crooks, R. N. (2013). The rainbow flag and the green carnation: Grindr in the gay village. First Monday, 18(11). Retrieved from http://www.firstmonday.dk/ojs/index. $\mathrm{php} / \mathrm{fm} / \mathrm{article} / \mathrm{view} / 4958 / 3790$.

David, G., \& Cambre, C. (2016). Screened intimacies: Tinder and the swipe logic. Social Media + Society, 2(2), 1-11.
Farman, J. (2012). Mobile interface theory: Embodied space and locative media. New York: Routledge.

Fincher, R., \& Jacobs, J. M. (Eds.). (1998). Cities of difference. New York: The Guilford Press.

Finn, E. (2017). What algorithms want: Imagination in the age of computing. Cambridge: The MIT Press.

Frith, J. (2017). Invisibility through the interface: The social consequences of spatial search. Media, Culture \& Society, 34(9), 536-551.

Hjorth, L., Pink, S., \& Horst, H. A. (2018). Being at home with privacy: Privacy and mundane intimacy through same-sex locative media practices. International Journal of Communication, 12, 1209-1227.

Hunte, B. (2020, June 1). Grindr removes "ethnicity filter” after complaints. BBC News. Retrieved from https://www.bbc.com/ news/technology-52886167.

Kitchin, R. (2014). The real-time city? Big data and smart urbanism. GeoJournal, 79(1), $1-14$.

Kitchin, R., \& Dodge, M. (2011). Code/space: Software and everyday life. Cambridge: The MIT Press.

Lefebvre, H. (1996). The specificity of the city (E. Kofman \& E. Lebas, Trans.). In E. Kofman \& E. Lebas (Eds.), Writings on cities (pp. 100-103). Oxford: Blackwell.

Leszczynski, A. (2015). Spatial media/tion. Progress in Human Geography, 39(6), 729-751.

Leszczynski, A. (2020). Glitchy vignettes of platform urbanism. Society and Space, 38(2), 189-208.

Licoppe, C. (2016). Mobilities and urban encounters in public places in the age of locative media: Seems, folds, and encounters with "pseudonymous strangers". Mobilities, 11(1), 99-116.

Mason, C. L. (2016). Tinder and humanitarian hook-ups: The erotics of social media racism. Feminist Media Studies, 16(5), 822-837.

McCullough, M. (2006). On the urbanism of locative media. Places, 18(2), 16-19.

Milgram, S. (1970). The experience of living in cities. Science, 167(3924), 1461-1468.

Pieber, D. A., \& Quan-Haase, A. (in press). Up close and impersonal: Locative media and the changing nature of the networked in- 
dividual in the city. In Z. Neal \& C. Rozenblat (Eds.). Handbook on cities and networks. Cheltenham: Edward Elgar.

Quan-Haase, A., \& Collins, J. L. (2008). I'm there, but I might not want to talk to you. Information, Communication \& Society, 11(4), 526-543.

Roth, Y. (2014). Locating the "Scruff guy": Theorizing body and space in gay geosocial media. International Journal of Communications, 8, 2113-2133.

Ryan, H. (2019). When Brooklyn was queer. New York: St. Martin's Press.

Simmel, G. (1922 [1955]). The web of group affiliations (R. Bendix, Trans.). In G. Simmel, Conflict and the web of group affiliations (pp. 125-195). Glencoe: Free Press.

Simmel, G. (1997). The sociology of space. In D. Frisby \& M. Featherstone (Eds.), Simmel on culture: Selected writings (pp. 137-169). London: Sage.

Sutko, D. M., \& de Souza e Silva, A. (2011). Location-aware mobile media and urban sociability. New Media \& Society, 13(5), 807-823.

Tonkiss, F. (2005). Space, the city and social theory: Social relations and urban forms. Cambridge: Polity.

Urry, J. (2007). Mobilities. Cambridge: Polity.
Veel, K., \& Thylstrup, N. B. (2018). Geolocating the stranger: The mapping of uncertainty as a configuration of matching and warranting techniques in dating apps. Journal of Aesthetics and Culture, 10(3), 43-52.

Villi, M. (2015). "Hey, I'm here Right Now": Camera phone photographs and mediated presence. Photographies, 8(1), 3-22.

Weber, M., Gerth, H., \& Mills, C. W. (1946). From Max Weber: Essays in sociology. New York: Oxford University Press.

Wellman, B., \& Leighton, B. (1979). Networks, neighborhoods and communities: Approaches to the community question. Urban Affairs, 14(3), 363-390.

Wilken, R. (2019). Cultural economies of locative media. New York: Oxford University Press.

Wilken, R., \& Goggin, G. (Eds.). (2015). Locative media. New York: Routledge.

Young, I. M. (1986). The ideal of community and the politics of difference. Social Theory and Practice, 12(1), 1-26.

Zerubavel, E. (1979). Private time and public time: The temporal structure of social accessibility and professional commitments. Social Forces, 58(1), 38-58. 